\title{
CHEMISTRY IN PLANETARY NEBULAE
}

\author{
P. COX \\ Institut d'Astrophysique Spatiale, Bât. 121 \\ Université de Paris XI, 91405 Orsay Cedex, France
}

\begin{abstract}
We review the properties of the molecular envelopes of planetary nebulae with an emphasis on the on-going chemistry which takes place in these regions.
\end{abstract}

\section{Introduction}

Recent observations have demonstrated that molecular gas is an important component of the planetary nebulae (PNe). Surveys have shown that the near-infrared 1-0 S(1) line of $\mathrm{H}_{2}$ (Kastner et al. 1996) and the millimeter lines of CO (Huggins et al. 1996) are readily detected in a large number (about 40) of $\mathrm{PNe}$, and several other species $\left(\mathrm{CN}, \mathrm{HCN}, \mathrm{HCO}^{+}\right)$have been detected and mapped in PNe including evolved ones (Cox et al. 1992, Bachiller et al. 1997). The molecular observations reveal that many PNe are surrounded by massive envelopes of neutral gas, and they provide a wealth of information on the physical state and chemistry of the gas, as well as on the overall kinematics of the envelope. In this paper we review recent advances in our understanding of the physics and chemistry characterizing the molecular envelopes of PNe.

\section{Results of $\mathrm{CO}$ and $\mathrm{H}_{2}$ surveys}

Huggins et al. (1996) reported a comprehensive survey of the millimeter $\mathrm{CO}$ emission in 91 planetary nebulae using the IRAM $30 \mathrm{~m}$ and SEST $15 \mathrm{~m}$ telescopes. These observations provide new detections and improved data for 23 nebulae in the $\mathrm{CO}(2-1)$ line, and sensitive limits for those not seen in CO. Including all these detections with additional detections reported in the literature (see Huggins et al. 1996 and references therein) $44 \mathrm{PNe}$ have now been detected in $\mathrm{CO}$ as compared to $80 \mathrm{PNe}$ for which upper limits or 


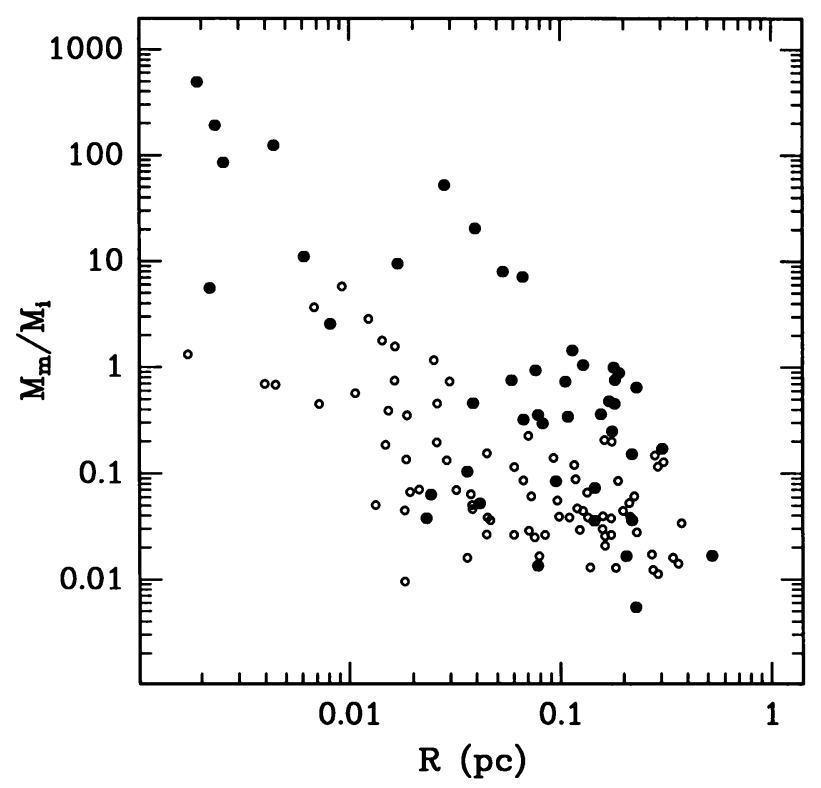

Figure 1. The molecular/ionized mass ratio versus nebular radius from the CO survey of planetary nebulae by Huggins et al. (1996). Filled symbols are detections, empty symbols are upper limits

non detections are reported. These results significantly extend the previous survey work by Huggins \& Healy (1989). The observations confirm the existence of an important class of PNe with substantial envelopes of molecular gas. They include little known PNe (e.g., M2-56, M3-3, He2-114), as well as familiar objects which have been well studied at other wavelengths (e.g., the Ring, the Dumbbell, the Helix, NGC 7027, NGC 2440). They range from compact to highly evolved $\mathrm{PNe}$, which implies that the molecular gas survives in the envelopes for a significant part of the evolution of the ionized gas. The $\mathrm{PNe}$ detected in $\mathrm{CO}$ typically have abundance ratios of $\mathrm{N} / \mathrm{O} \geq 0.3$. Their morphology is elliptical or bipolar (with a waist), indicating evolution from the more massive PN precursors of a young disk population. A similar finding has been reported by Kastner et al. (1996): from the imaging survey of the $2.12 \mu \mathrm{m} \mathrm{H}_{2}$ emission from $\sim 60 \mathrm{PNe}$, they concluded that all the $\mathrm{PNe}$ which are detected also possess bipolar morphology.

The masses of the molecular envelopes estimated for the detected $\mathrm{PNe}$ are typically in the range $10^{-2}$ to a few $\mathrm{M}_{\odot}$, and in more than a dozen cases they exceed the mass of the ionized nebula $\left(M_{i}\right)$ determined from the radio continuum. The column density of molecular gas through the envelopes and their mass relative to the mass of ionized gas show dramatic decreases with increasing nebular size, documenting the expansion of the envelopes and 
the growth of the optical nebulae at the expense of the molecular gas. This is illustrated in Fig. 1 which shows the mass ratio $M_{m} / M_{i}$ versus the radius of the ionized nebulae $R$, which is roughly an indicator of the age of PNe: the range $R \sim 0.003-0.3 \mathrm{pc}$ corresponds to expansion timescales of $\sim 100$ - 10,000 yr. The molecular envelopes remain a major mass component in the objects until the nebulae reach a radius of about $0.1 \mathrm{pc}$.

The nebulae not detected in $\mathrm{CO}$ have little or no molecular gas $\left(10^{-3}\right.$ to $10^{-2} M_{\odot}$ ), and in many cases their envelopes must be rapidly photodissociated before or during the compact phase. The large differences in the molecular gas content of the nebulae highlight the different evolutionary paths for $\mathrm{PNe}$ formation which result from the range in mass of the progenitor stars and the structure of their circumstellar envelopes.

\section{Clumpiness}

The extreme conditions characterizing the transition from the AGB to the PN phase - very strong ultraviolet radiation fields from the evolving central stars and violent shocks generated by fast stellar winds - imply that the properties of the PNe molecular envelopes must be well adapted to survive over the dynamical lifetime of the nebulae (about 10,000 years). The clumping of the molecular gas in PNe is an essential aspect of both their physical and chemical evolution as noted by Tielens (1993). From detailed CO mapping results (e.g., Bachiller et al. 1993), it is now known that the massive envelopes of molecular gas which surround the $\mathrm{PNe}$ are highly fragmented, spheroidal shells or rings around the ionized nebulae. Rather than being homogeneous, PNe envelopes consist of a collection of numerous, dense and small globules expanding together with the ionized gas in which they are immersed.

The best case study of clumpiness inside the envelope of a PN is the Helix nebula, one of the most familiar planetary nebulae, which is often considered as the prototype of evolved PNe. At a distance of $130 \mathrm{pc}$, it is the closest PN and is far larger in angular size than any other nebula. The Helix nebula thus affords the opportunity to explore its large and small scale stucture in great detail. The Helix nebula contains at least $0.03 \mathrm{M}_{\odot}$ of molecular gas, or $25 \%$ of the total nebular mass (Huggins \& Healy 1986). The molecular gas forms a large incomplete shell structure which breaks down in numerous sub-structures (Forveille \& Huggins 1991, Cox et al. in preparation). Huggins et al. (1992) have shown that the molecular gas has managed to survive in the cometary globules which are embedded within the ionized cavity. These small $(\sim 0.001 \mathrm{pc})$ globules consist of dense condensations of molecular gas with typical masses $\left(\geq 510^{-6} \mathrm{M}_{\odot}\right)$ and densities $\left(\geq 10^{5} \mathrm{~cm}^{-3}\right)$. Similar numbers have been derived for these globules from 

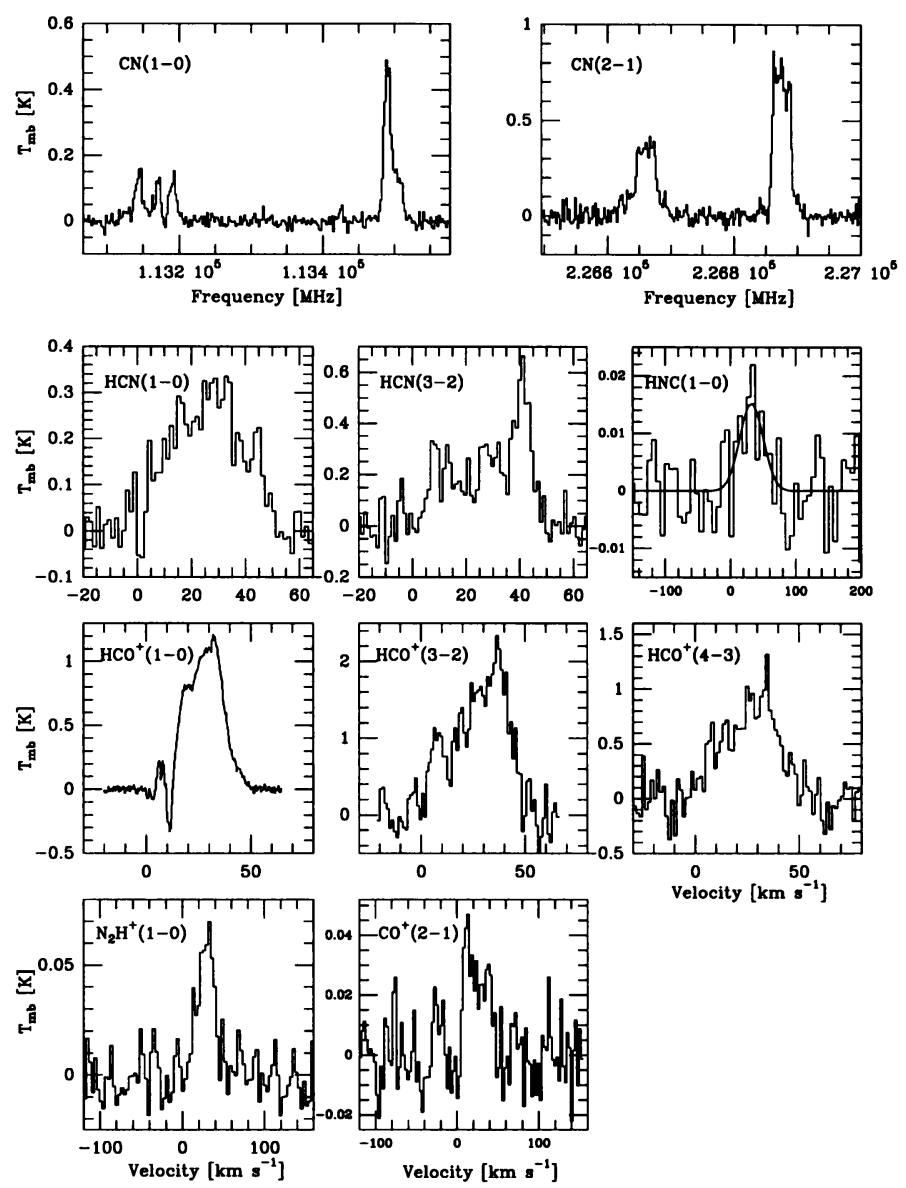

Figure 2. Molecular species (other than CO and its isotopes) detected in NGC 7027. All measurements are from the IRAM 30-meter telescope except $\mathrm{HCO}^{+}(4-3)$ which was observed with the CSO 10-meter telescope - from Cox et al. (in preparation).

the spectacular HST imaging data published by O'Dell \& Handron (1996).

As shown by Bachiller et al. (1997), the volume density of the molecular clumps in PNe does not change significantly with nebular age and has a typical value of a few $\times 10^{5} \mathrm{~cm}^{-3}$. Dense clumps can thus survive for much of the nebular evolution with essentially constant density. Clumpiness is an essential ingredient of the PN envelopes that determines their physical and chemical evolution throughout their lifetime. The neutral envelopes of $\mathrm{PNe}$ are thus extreme cases of dense photo-dissociation regions (PDRs) and their chemical composition is expected to be quite different from that in interstellar clouds or circumstellar envelopes of AGB stars. 


\section{Chemistry}

In spite of the intrinsic interest of the chemistry in $\mathrm{PNe}$, observations of the chemical composition of the molecular gas have been largely confined to the study of the massive envelope of NGC 7027 because of the weakness of the lines in other envelopes. In addition to CO, NGC 7027 has been detected in lines of HCN (Olofsson et al. 1982), CN (Thronson \& Bally 1986), $\mathrm{C}_{3} \mathrm{H}_{2}$ (Cox et al. 1987), $\mathrm{HCO}^{+}$(Deguchi et al. 1990), $\mathrm{HNC}$ and $\mathrm{N}_{2} \mathrm{H}^{+}$(Cox et al. 1993), and $\mathrm{CO}^{+}$(Latter et al. 1993). Fig. 2 displays the spectra of these molecules in various transitions. In other PNe, HCN, HNC, and $\mathrm{HCO}^{+}$have been detected in NGC 2346 by Bachiller et al. (1989) and also in NGC 6072 and IC 4406 together with strong CN emission by Cox et al. (1992) and in M1-16 by Sahai et al. (1994). These observations already indicated that the molecular abundances are indeed unusual. This trend has been confirmed by a recent search for line emission of nine molecular species other than CO in a sample of PNe by Bachiller et al. (1997). The sample represents different evolutionary stages and includes the proto-PNe CRL 618 and CRL 2688, the young PN NGC 7027, and the evolved PNe NGC 6720 (the Ring), M4-9, NGC 6781, and NGC 7293 (the Helix). These results provide the basis for a systematic study of the changes in chemistry of the envelopes along the evolutionary sequence from AGB to PN.

Figure 3 shows how the $\mathrm{CN}, \mathrm{HCN}, \mathrm{HNC}, \mathrm{HCO}^{+}$, and $\mathrm{CS}$ abundances vary among the observed sources, which have been ordered according to their degree of evolution. The left hand axis indicates abundances relative to ${ }^{13} \mathrm{CO}$, and the right hand axis indicates abundances relative to $\mathrm{H}_{2}$. Fig. 3 indicates that significant changes in the molecular abundances occur during the evolution of the envelopes. The largest effect is the rapid increase in the abundance of $\mathrm{HCO}^{+}$by about two orders of magnitude during the proto-PN to $\mathrm{PN}$ transition. The $\mathrm{CN}$ abundance also increases but by a smaller factor of about 4 . At the same time, the species $\mathrm{CS}, \mathrm{SiO}, \mathrm{SiC}_{2}$ and $\mathrm{HC}_{3} \mathrm{~N}$ decrease and must be effectively destroyed when the planetary nebula forms. During the PN phase, the abundances of the detected molecules remain essentially the same even though they span a large range in evolution. HCN, HNC and $\mathrm{HCO}^{+}$have comparable abundances, but $\mathrm{CN}$ is about an order of magnitude more abundant. On average, Bachiller et al. (1997) find that $\mathrm{CN} / \mathrm{HCN}=9.0, \mathrm{HNC} / \mathrm{HCN}=0.45$, and $\mathrm{HCO}^{+} / \mathrm{HCN}=0.54$ in evolved $\mathrm{PNe}$. These ratios are enhanced by one, two, and three orders of magnitude, respectively, over the values observed in the prototypical AGB envelope IRC+10216 (Glassgold 1996).

The main processes which determine the chemical evolution of the molecular envelopes of PNe have been discussed in Cox et al. (1992). The essential influence on the chemistry is the ultraviolet radiation field of the central 


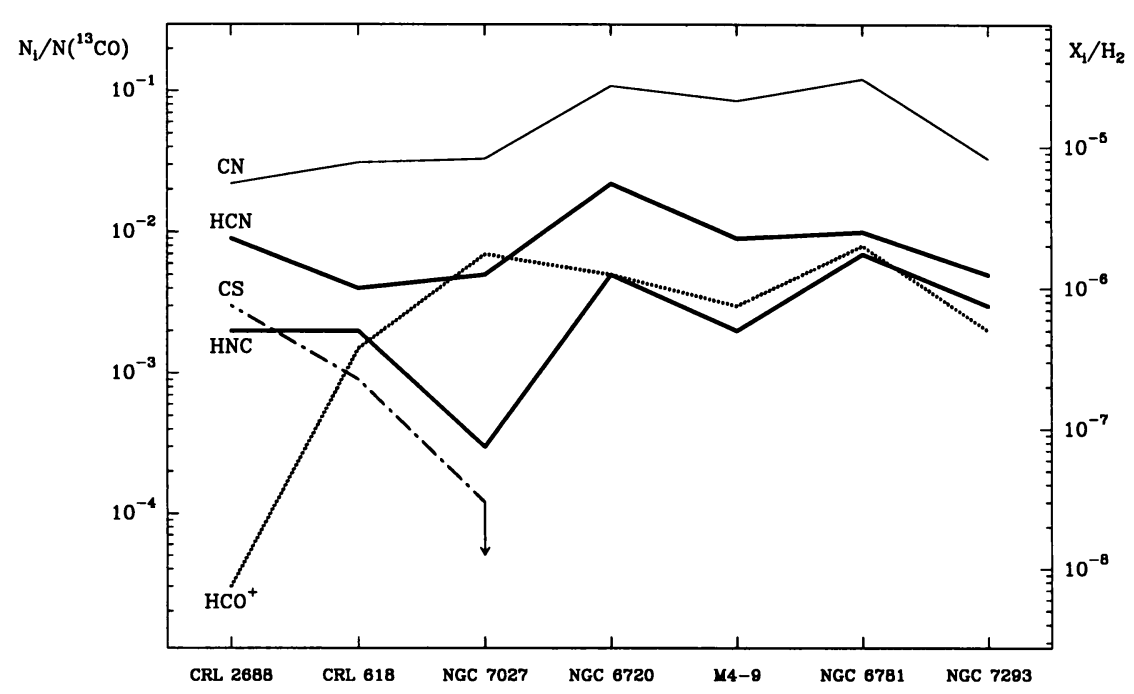

Figure 3. Molecular abundances in proto-planetary nebulae and planetary nebulae, relative to ${ }^{13} \mathrm{CO}$. The scale on the right vertical axis indicates the abundances relative to $\mathrm{H}_{2}$, for an assumed ${ }^{13} \mathrm{CO}$ abundance of $2.5 \times 10^{-5}$. The variation of the $\mathrm{CS}$ abundance is representative of that of $\mathrm{HC}_{3} \mathrm{~N}, \mathrm{SiO}$, and $\mathrm{SiC}_{2}$ which are only detected in the proto-PNe - from Bachiller et al. (1997)

star. During the rapid transition from proto-PN to PN, the ultraviolet radiation field incident on the inner surface of the molecular envelope rises to very high values $\left(\chi \sim 10^{5}\right.$ in units of the average interstellar radiation field) and then gradually decreases because of the expansion of the envelope and the decrease in luminosity of the central star $(\chi \sim 10-100)$. This gives rise to extreme PDRs in the neutral gas (Tielens 1993, Hollenbach \& Natta 1995) which are characterized by fast evolution unlike those in interstellar clouds (e.g., Tielens \& Hollenbach 1985). Photo-dissociation and a high level of ionization (including perhaps the X-ray emission from the hot central star in the young PN stage) are likely the main drivers of the chemistry after the proto-PN phase, although no detailed thermal-chemical models have yet been developed for carbon-rich (or oxygen-rich) envelopes of PNe. PDRs have been detected in the envelopes of PNe through the emission of atomic fine structure lines, e.g., in the far-infrared for the [CII] and [OI] lines (using the KAO - Dinerstein et al. 1995, Young et al. 1997a; and more recently with ISO - Liu et al. 1996), and, in the submillimeter, with the ground state of CI which has been detected in NGC 7027 (Young et al. 1997a), and in the more evolved Ring (Bachiller et al. 1994) and 
Helix (Young et al. 1997b) nebulae. In these hostile environments, the survival of $\mathrm{CO}$ and the less abundant molecular species such as $\mathrm{CN}, \mathrm{HCN}$ and $\mathrm{HCO}^{+}$is entirely due to the presence of high-density gas which is found in the form of small clumps. In an homogeneous, expanding envelope, the gas becomes optically thin and diffuse on short timescales, and is rapidly photo-dissociated (typically in a few hundred years).

Besides the ultraviolet radiation field, the effect of shocks might play an important rôle in the early stages of the proto-PN phase when the fast stellar winds compress and heat the inner surface of the molecular envelope making some endothermic reactions possible. However, the importance of shocks in the chemistry of $\mathrm{PNe}$ is difficult to evaluate because the dynamical timescale of the gas is not known in detail. High-velocity winds are observed in proto-PN (CRL 618 and CRL 2688) and in some young PNe but the winds are mainly confined in the polar directions of these bipolar nebulae and affect only marginally the bulk of the gas in the denser equatorial waist. Howe et al. (1992) modelled the shock chemistry in PNe in the frame of the two-wind scenario and find that the reformation of molecules in shocked gas leads to the synthesis of small molecules and ions such as $\mathrm{CH}, \mathrm{CH}_{2}$, and $\mathrm{CH}_{3}^{+}$but not the heavier species reported in Bachiller et al. (1997). Further studies of the chemistry induced by shocks under different conditions, in particular in the presence of dense clumps, remains to be done.

The large abundance of $\mathrm{CN}$ which is observed in PNe is entirely consistent with the effects of chemistry dominated by ultraviolet radiation. The CN in AGB envelopes is produced in the outer regions through the HCN photo-destruction by ambient ultraviolet radiation (Glassgold 1996). As the stellar radiation turns on inside, a general increase in the $\mathrm{CN} / \mathrm{HCN}$ ratio will occur as a result of the photodissociation and photoionization of the HCN-rich inner envelope and locally as a result of the smaller effective photo-dissociation rate for $\mathrm{CN}$. The models developed by Howe et al. (1994) for the globules in the Helix nebula lend support to the above picture and their predicted abundances for $\mathrm{CN}$ are comparable to the abundances reported in Bachiller et al. (1997). HNC is formed in carbon-rich AGB envelopes and in the interstellar medium from dissociative recombination of $\mathrm{HCNH}^{+}$(Glassgold 1996 and references therein). A similar mechanism is likely at play in PNe. The HNC/HCN abundance ratio predicted in the globule model of Howe et al. (1994) is a factor 20 lower than observed but could possibly be reconciled with a change in some of the model parameters. The very low HNC/HCN ratio found for NGC 7027 could be the result of the high temperatures in the gas of this young PN (Cox et al. in preparation)

The large abundances derived for $\mathrm{HCO}^{+}$which are found just after the onset of the PN stage and persist throughout the nebular evolution is the 
most difficult observation to explain. The problem was first recognized by Deguchi et al. (1992) and Cox et al. (1992) in NGC 7027 and in the PNe NGC 6072 and IC 4406, respectively. The problem is equally apparent in the Helix nebula where the predictions of the $\mathrm{HCO}^{+}$abundance by Howe et al. (1994) are about three orders of magnitude lower than observed. $\mathrm{HCO}^{+}$ is probably formed in PNe by gas-phase reactions as in the interstellar medium, with the difference that the $\mathrm{H}_{3}^{+}$abundance in $\mathrm{PNe}$ is low due to dissociative recombination so that a large ionization rate of $\mathrm{H}_{2}$ is needed. Deguchi et al. (1992) suggested that X-rays from the hot central star might play an important rôle. This could explain the high $\mathrm{HCO}^{+}$abundance in NGC 7027. But this explanation is at fault for the more evolved objects (such as the Helix) where similar amounts of $\mathrm{HCO}^{+}$are observed whereas the X-ray flux from the central star is lower by several orders of magnitude as compared to NGC 7027 (see also Cox et al. 1992). The large ultraviolet radiation and the clumpy structure of the PDR gas are likely key ingredients in the chemistry of $\mathrm{HCO}^{+}$in $\mathrm{PNe}$, although the influence of shocks and the subsequent endothermic reactions are also possible.

Finally, the complexity of the chemistry in PNe is underscored by the recent unexpected detection with ISO of water vapour and $\mathrm{OH}$ in the carbonrich envelope of NGC 7027 (Liu et al. 1996; Cox, this volume). The most likely explanation is that these species originate in the warm and dense molecular gas of the PDR, although the detailed chemistry remains to be explored. The formation of $\mathrm{H}_{2} \mathrm{O}$ and $\mathrm{OH}$ could well be related to the large abundance of $\mathrm{HCO}^{+}$and to the rich on-going ion-chemistry which takes place in the inner parts of this young planetary nebula.

\section{Conclusions}

The above results demonstrate that the recent increase in sensitivity and spatial resolution in the millimeter have been essential in the study of the physical and chemical properties of the molecular envelopes of PNe throughout their evolution. The massive molecular envelopes of PNe are subject to the intense ultraviolet radiation field of their hot central stars. The presence of an active on-going ion-chemistry has been documented by recent survey work and shows that a number of molecular species can survive in $\mathrm{PNe}$ to advanced stages of evolution. The fragmented structure of the envelopes is essential to protect the molecular species from photo-destruction. The densities of the clumps of gas are typically of the order a few $10^{5} \mathrm{~cm}^{-3}$ with temperatures of about $25 \mathrm{~K}$, consistent with the idea that clumps are roughly in pressure equilibrium with the ionized gas. The molecular abundances in PNe differ significantly from those of the precursor AGB envelope and the interstellar medium. Whereas most of the abundances are 
well explained by the influence of the ultraviolet radiation field, abundances of some species (most notably $\mathrm{HCO}^{+}$) are still not understood although the rôle of X-rays and/or shocks has been suggested. This underscores the fact that the physics and chemistry of the unusual photodissociation regions which are created in the envelopes surrounding the hot central stars of planetary nebulae should further be explored both observationally and theoretically.

\section{Acknowledgements}

It is a great pleasure to thank colleagues Drs. P.J. Huggins, R. Bachiller, and T. Forveille: the results of our collaborative programs form the basis of this paper.

\section{References}

Bachiller, R., Bujarrabal, V., Martín-Pintado, J., Gomez-Gonzalez, J. 1989, A\&A 218, 252

Bachiller, R., Huggins, P.J., Cox, P., Forveille, T. 1993, A\&A 267, 177

Bachiller, R., Huggins, P.J., Cox, P., Forveille, T. 1994, A\&A 281, L93

Bachiller, R., Forveille, T., Huggins, P.J., Cox, P., Omont, A. 1997, A\&A, in press

Cox, P., Güsten, R., Henkel, H. 1987, A\&A 181, L19

Cox, P., Omont, A., Huggins, P.J., Bachiller, R., Forveille, T. 1992, A\&A 266, 420

Cox, P. et al. 1993, in Planetary Nebulae, Weinberger R. \& Acker A. (eds.) (Kluwer, Dordrecht), p. 227

Deguchi, D., Izumiura, H., Kaifu, N., Moa, X., Nguyen-Q-Rieu, Ukita, N. 1990 ApJ 351,522

Dinerstein, H., Haas, M., Erickson, E.F., Werner, M.W. 1995, in Airborne Astronomy Symposium on the Galactic Ecosystem, M.R. Haas, J.A. Davidson, and E.F. Erickson (eds.) ASP Conference Series, vol. 73, p. 387

Forveille, T., Huggins, P.J. 1991, A\&A 248, 599

Glassgold, A.E. 1996, ARAA 34, 241

Hollenbach, D.J., Natta, A. 1995, ApJ 455, 133

Howe, D.A., Millar, T.J., Williams, D.A. 1992, MNRAS 255, 217

Howe, D.A., Hartquist, T.W., Williams , D.A. 1994, MNRAS 271, 811

Huggins, P.J., Healy, A.P. 1986, ApJ 305, L29

Huggins, P.J., Healy, A.P. 1989, ApJ 346, 201

Huggins, P.J., Bachiller, R., Cox, P., Forveille, T. 1992, ApJ 401, L43

Huggins, P.J. 1993, in Planetary Nebulae, Weinberger R. \& Acker A. (eds.) (Kluwer, Dordrecht), p. 147

Huggins, P.J., Bachiller, R., Cox, P., Forveille, T. 1996, A\&A 315, 284

Kastner, J.H., Weintraub, D.A., Gatley, I., Merrill, K.M., Probst, R.G. 1996, ApJ 462, 777

Latter, W.B., Walker, C.K., Maloney, P.R 1993, ApJ 419, L97

Liu, X.-W. et al. 1996, A\&A 315, L257

O'Dell, C.R., Handron, K.D. 1996, AJ 111, 1630

Olofsson, H., Johansson, L., Nguyen-Q-Rieu, Sopka, B., Zuckerman, B. 1982 BAAS 14,895

Sahai, R., Wooten, A., Schwarz, H., Wild, W. 1994 ApJ 428, 237

Thronson, H.A., Bally, J. 1986, ApJ 300, 749 
Tielens, A.G.G.M., Hollenbach, D.J. 1985, ApJ 291, 722

Tielens, A.G.G.M. 1993, in Planetary Nebulae, Weinberger R. \& Acker A. (eds.) (Kluwer, Dordrecht), p. 155

Young, K., Keene, J., Phillips, T.G., Betz, A.L., Boreiko, R.T. 1997a, ApJ submitted

Young, K., Cox, P., Huggins, P.J., Forveille, T., Bachiller, R. 1997b, ApJ submitted 